\title{
Modeling secondary organic aerosol formation through cloud processing of organic compounds
}

\author{
J. Chen ${ }^{1}$, R. J. Griffin ${ }^{1,2}$, A. Grini ${ }^{3}$, and P. Tulet ${ }^{4}$ \\ ${ }^{1}$ Institute for the Study of Earth, Oceans, and Space, University of New Hampshire, Durham, NH, USA \\ ${ }^{2}$ Department of Earth Sciences, University of New Hampshire, Durham, NH, USA \\ ${ }^{3}$ Department of Geoscience, University of Oslo, Pb 1022 Blindern, 0315, Oslo, Norway \\ ${ }^{4}$ CNRM/GMEI Météo France, Toulouse, France
}

Received: 30 May 2007 - Published in Atmos. Chem. Phys. Discuss.: 26 June 2007

Revised: 3 September 2007 - Accepted: 5 October 2007 - Published: 17 October 2007

\begin{abstract}
Interest in the potential formation of secondary organic aerosol (SOA) through reactions of organic compounds in condensed aqueous phases is growing. In this study, the potential formation of SOA from irreversible aqueousphase reactions of organic species in clouds was investigated. A new proposed aqueous-phase chemistry mechanism (AqChem) is coupled with the existing gas-phase Caltech Atmospheric Chemistry Mechanism (CACM) and the Model to Predict the Multiphase Partitioning of Organics (MPMPO) that simulate SOA formation. AqChem treats irreversible organic reactions that lead mainly to the formation of carboxylic acids, which are usually less volatile than the corresponding aldehydic compounds. Zero-dimensional model simulations were performed for tropospheric conditions with clouds present for three consecutive hours per day. Zero-dimensional model simulations show that 48-h average SOA formation is increased by $27 \%$ for a rural scenario with strong monoterpene emissions and $7 \%$ for an urban scenario with strong emissions of aromatic compounds, respectively, when irreversible organic reactions in clouds are considered. AqChem was also incorporated into the Community Multiscale Air Quality Model (CMAQ) version 4.4 with CACM/MPMPO and applied to a previously studied photochemical episode (3-4 August 2004) focusing on the eastern United States. The CMAQ study indicates that the maximum contribution of SOA formation from irreversible reactions of organics in clouds is $0.28 \mu \mathrm{g} \mathrm{m}^{-3}$ for 24 -h average concentrations and $0.60 \mu \mathrm{g} \mathrm{m}^{-3}$ for one-hour average concentrations at certain locations. On average, domain-wide surface SOA predictions for the episode are increased by $9 \%$ when irreversible, in-cloud processing of organics is considered. Because aldehydes of carbon number greater than four are assumed to convert fully to the corresponding carboxylic
\end{abstract}

Correspondence to: J. Chen

(jianjunc@alumni.unh.edu) acids upon reaction with $\mathrm{OH}$ in cloud droplets and this assumption may overestimate carboxylic acid formation from this reaction route, the present study provides an upper bound estimate of SOA formation via this pathway.

\section{Introduction}

Atmospheric particulate matter (PM) is associated with adverse human health effects (Pope and Dockery, 2006), decreases in visibility (Malm, 1989), and global climate forcing (Charlson et al., 1992; Jones et al., 1994). Organic aerosol (OA) is a ubiquitous and important constituent of atmospheric PM (Murphy et al., 1998; Turpin et al., 2000). OA consists of primary OA (POA), which is emitted directly from various sources, and secondary OA (SOA), which is formed in the atmosphere from the oxidation of volatile organic compounds (VOCs).

The processes leading to SOA formation typically are viewed as gas-phase oxidation of VOCs followed by nucleation/gas-particle partitioning of low-volatility products (Pankow, 1994; Odum et al., 1996; Griffin et al., 1999). Oligomer and/or polymer formation in the aerosol phase also has been shown to be important with regard to SOA formation (Jang et al., 2002; Kalberer et al., 2004). Besides these processes, it also has been hypothesized that fog/cloud processing, which leads to substantial sulfate formation, could also be a source for SOA (Blando and Turpin, 2000). The process of SOA formation from aqueous-phase processing of organic compounds in clouds involves formation of potential low-volatility products through aqueous-phase reactions of organics, followed by subsequent gas-particle partitioning of the low-volatility products post evaporation of the hydrometeor (Blando and Turpin, 2000; Kanakidou et al., 2005). Recent experimental and modeling studies indeed demonstrated

Published by Copernicus Publications on behalf of the European Geosciences Union. 


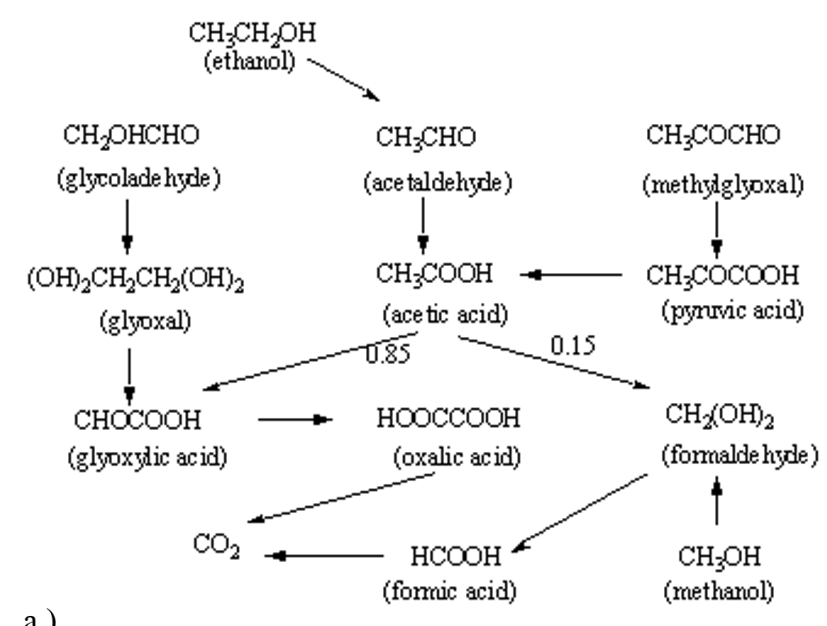

a.)

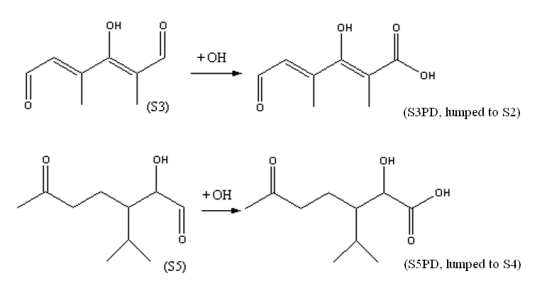

b.)
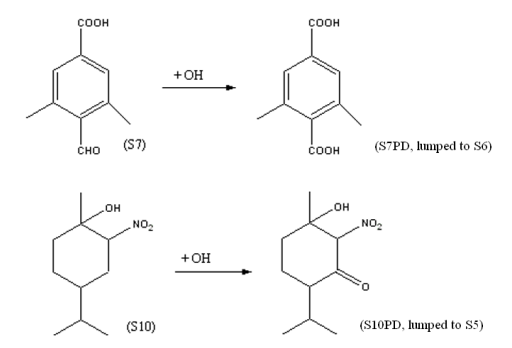

Fig. 1. Schematic of organic reactions in clouds: (a) organics of carbon number less than four (based on Ervens et al., 2004 and Lim et al., 2005); (b) organics of carbon number greater than four (based on Aumont et al., 2000) (S2-S7 and S10 are surrogates in the MPMPO module).

that SOA potentially can be formed from aqueous-phase processing of organic compounds in clouds (Altieri et al., 2006; Carlton et al., 2006; Loeffler et al., 2006). Ambient particle size distribution measurements also showed the occurrence of droplet-mode organics (Blando et al., 1998; Yao et al., 2002), which, similarly to droplet-mode sulfate, most likely are formed from cloud processing of organic materials (Blando and Turpin, 2000). Several modeling studies (Warneck, 2003; Ervens et al., 2004; Lim et al., 2005; Sorooshian et al., 2006; Sorooshian et al., 2007) have simulated successfully the formation of low-molecular weight dicarboxylic acid from cloud processing of organics. Such dicarboxylic acids have been found in atmospheric aerosols in various regions (Kawamura and Ikushima, 1993; Decesari et al., 2000). In addition, Claeys et al. (2004) showed that multiphase acid- catalyzed organic reactions with hydrogen peroxide provided a new route for SOA formation from isoprene and hypothesized that such a mechanism also could provide a pathway for SOA formation from monoterpenes and their oxidation products. Most recently, Heald et al. (2006) analyzed the covariance of water soluble particulate organics with other species in the free troposphere over the eastern United States, with the results suggesting aqueous-phase SOA generation involving biogenic precursors.

Given the increasing interest in potential SOA formation through the aqueous-phase processing of organic compounds, this paper evaluates the significance of SOA formation from aqueous-phase reactions of organics in cloud droplets through a modeling study. The study involves the development of an aqueous-phase chemistry mechanism (AqChem) that specifically includes treatment of potential organic reactions that lead to production of semi-volatile organic compounds. Because of our incomplete knowledge on the aqueous-phase chemistry for organics (Kanakidou et al., 2005; Gelencser and Varga, 2005), AqChem treats organic reactions based on previous work by Ervens et al. (2004) and Lim et al. (2005) for organics of carbon number up to four and the simple protocol used by Aumont et al. (2000) for organics of carbon number greater than four. Potential reversible oligomerization reactions (Altieri et al., 2006) are not included because of a current lack of a quantitative description of these phenomena. AqChem utilizes an existing gas-phase chemistry mechanism, the Caltech Atmospheric Chemistry Mechanism (CACM) (Griffin et al., 2002) that provides the prediction of gas-phase production of organic compounds. It is also linked with a SOA module, the Model to Predict the Multi-phase Partitioning of Organics (MPMPO) (Griffin et al., 2003), such that the partitioning between the gas and aerosol phases of semi-volatile organic products from aqueous-phase reactions is determined thermodynamically. AqChem was used in a zero-dimensional model as well as a three-dimensional model to study the magnitude of potential SOA formation in clouds.

\section{Methods}

\subsection{Development of the AqChem mechanism}

AqChem was developed based on previous work (Aumont et al., 2000; Warneck, 2003; Ervens et al., 2004; Lim et al., 2005) and to work specifically with the CACM mechanism and the MPMPO module. The main purpose of AqChem is to treat the irreversible organic reactions that potentially lead to the production of semi-volatile organic compounds in clouds.

In AqChem, the reactions for organics of carbon number up to four that are treated in the CACM mechanism were adopted from previous work by Ervens et al. (2004) and Lim et al. (2005) and are shown schematically in Fig. 1a. The 
main purpose of the reaction scheme for organics of carbon number up to four is to provide formation routes for oxalic and pyruvic acids. Briefly, organics in the cloud phase are assumed to be oxidized only by hydroxyl radical $(\mathrm{OH})$. Glycoaldehyde and glyoxal are converted to oxalic acid via glyoxylic acid (Ervens et al., 2004; Lim et al., 2005). Methylglyoxal is converted to pyruvic acid, which decomposes to form acetic acid. Ethanol and acetaldehyde are converted to acetic acid as well. A portion of acetic acid is converted to oxalic acid, while the rest decomposes to formaldehyde (Lim et al., 2005). No reactions are treated for methyl vinyl ketone and methacrolein in the aqueous phase because of their low solubility in water (Ervens et al., 2004).

Within CACM, there are approximately 140 semi-volatile organic compounds of carbon number greater than four (Griffin et al., 2002; Chen and Griffin, 2005). Instead of treating potential aqueous-phase chemistry for these organic compounds individually, an organic surrogate approach similar to that used in MPMPO was adopted here. In the MPMPO module, each semi-volatile organic compound is lumped into one of 11 organic surrogate species (Griffin et al., 2003; Griffin et al., 2005; Chen et al., 2006). MPMPO calculates the partitioning between the gas and aerosol phases for these 11 surrogate species. Similarly, AqChem treats the aqueousphase chemistry for four of the 11 surrogates because of their potential to lead to less volatile organic products (e.g., carboxylic acids). These four surrogate species are surrogate 3 (S3, 3-hydroxy-2, 4-dimethyl-2, 4-hexadiendial), surrogate 5 (S5, 2-hydroxyl-3-isopropyl-6-keto-heptanal), surrogate 7 (S7, 2,4-dimethyl-3-formyl-benzoic acid), and surrogate 10 (S10, 1-methyl-1-hydroxy-2-nitrato-4-isopropylcyclohexane) in the MPMPO module (Griffin et al., 2003; Griffin et al., 2005). In this way, although only four aqueousphase chemical reactions were added for organic compounds of carbon number greater than four, 58 organic compounds that are lumped into these four surrogates in the MPMPO undergo further reactions in the aqueous phase. The products of aqueous-phase reactions for these four MPMPO surrogates were based on the protocol used by Aumont et al. (2000). Aumont et al. (2000) assumed that (1) $\mathrm{OH}$ is the only oxidant; (2) an aldehyde moiety is converted to a carboxylic acid moiety; and (3) the carbon skeleton is not changed during oxidation. Following the protocol of Aumont et al. (2000), the oxidation of $\mathrm{S} 3$ by $\mathrm{OH}$ leads to the formation of 3hydroxy-2, 4-dimethyl-2, 4-hexadienalic acid (S3PD). The oxidation product of S5 is 2-hydroxyl-3-isopropyl-6-ketoheptanoic acid (S5PD). S7 is converted to 3, 5-dimethyl-1, 4dibenzoic acid (S7PD). Oxidation of $\mathrm{S} 10$ by $\mathrm{OH}$ in the aqueous phase leads to a product (S10PD, 1-methyl-1-hydroxy2-nitrato-3-oxo-4-isopropyl-cyclohexane) that has one more ketone group than surrogate 10. The assumption of full conversion of aldehydes of carbon number greater than four to their corresponding acids likely represents an upper-bound estimate of the yields of carboxylic acids from this reaction pathway (Carlton et al., 2006). The aqueous-phase oxidation

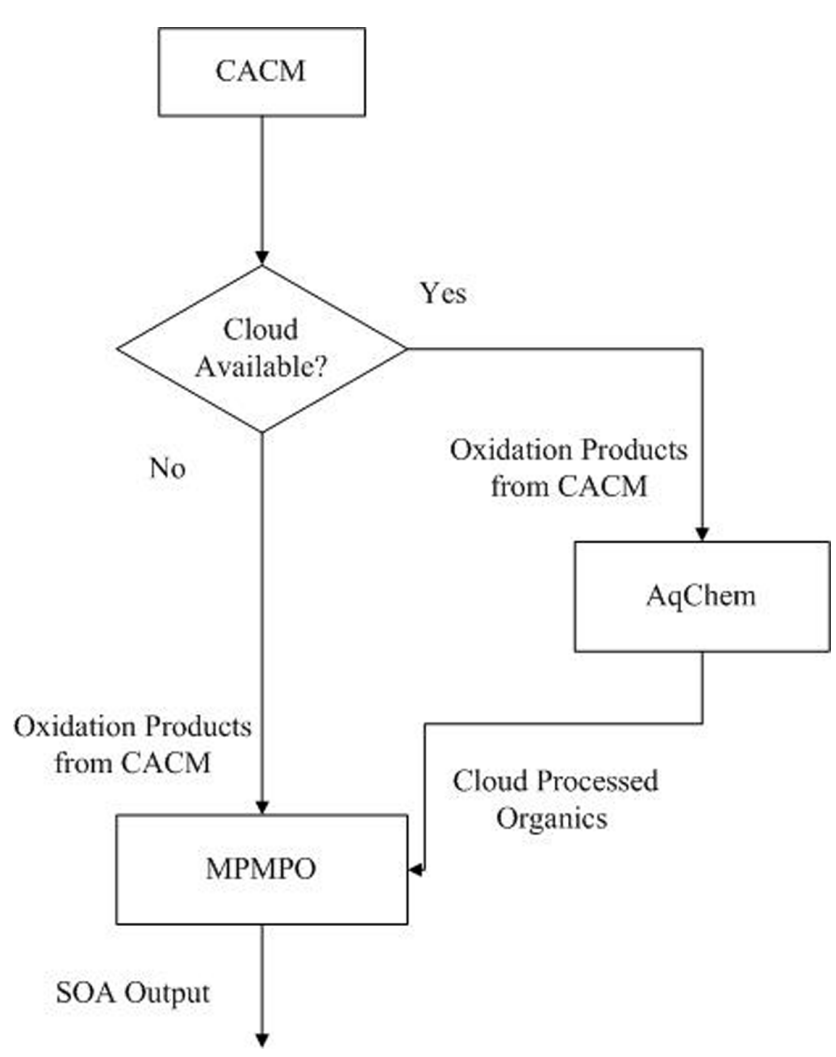

Fig. 2. Flow diagram for SOA modeling with/without consideration of the aqueous-phase chemistry in clouds.

rates by $\mathrm{OH}$ for these surrogate species were estimated based on the method of Dutot et al. (2003). The reaction scheme for these surrogate species is shown in Fig. $1 \mathrm{~b}$.

AqChem also includes a compact treatment of aqueousphase hydrogen-oxygen and sulfur chemistry mainly based on Ervens et al. (2003) and Ervens et al. (2004). Such a compact treatment includes the important reactions for determining aqueous $\mathrm{OH}$ levels and aqueous sulfate formation but is computationally less demanding than detailed treatments, which is especially important for three-dimensional air quality simulations. Tables 1 and 2 list the irreversible and equilibrium reactions, respectively, that are included in AqChem. Gas and aqueous-phase mass transfer was treated according to the resistance model of Schwartz (1986) by considering mass accommodation coefficient $(\alpha)$, gas-phase diffusion constants $\left(D_{g}\right)$, and Henry's law constants $\left(K_{H}\right)$ for each soluble species, values of which are shown in Table 3.

After the evaporation of cloud droplets, the partitioning between the gas and aerosol phases for the oxidation products formed from the aqueous-phase reactions is computed by the MPMPO module. This is shown schematically in Fig. 2. If clouds are not present, the amounts of semi-volatile organic compounds computed from the CACM mechanism are used directly as input to the MPMPO module to compute SOA formation, as in previous applications (Chen et 
Table 1. Aqueous-phase irreversible reactions included in AqChem.

\begin{tabular}{|c|c|c|c|c|}
\hline Num. & Reactions & $\mathrm{k}^{\mathrm{a}}$ & $\mathrm{E} / \mathrm{R}(\mathrm{K})$ & Ref. $^{b}$ \\
\hline 1 & $\mathrm{O}_{3}+\mathrm{O}_{2}^{-} \rightarrow 2 \mathrm{O}_{2}+\mathrm{OH}+\mathrm{OH}^{-}$ & $1.5 \mathrm{e} 9$ & 2200 & 2 \\
\hline 2 & $\mathrm{H}_{2} \mathrm{O}_{2}+\mathrm{OH} \rightarrow \mathrm{HO}_{2}+\mathrm{H}_{2} \mathrm{O}$ & $3.0 \mathrm{e} 7$ & 1680 & 1 \\
\hline $3^{\mathrm{c}}$ & $\mathrm{H}_{2} \mathrm{O}_{2}+\mathrm{hv} \rightarrow 2 \mathrm{OH}$ & & & 1 \\
\hline 4 & $\mathrm{HO}_{2}+\mathrm{O}_{2}^{-} \rightarrow \mathrm{H}_{2} \mathrm{O}_{2}+\mathrm{O}_{2}+\mathrm{OH}^{-}$ & $9.7 \mathrm{e} 7$ & 1060 & 1 \\
\hline 5 & $\mathrm{HO}_{2}+\mathrm{HO}_{2} \rightarrow \mathrm{H}_{2} \mathrm{O}_{2}+\mathrm{O}_{2}$ & $8.3 \mathrm{e} 5$ & 2720 & 1 \\
\hline 6 & $\mathrm{OH}+\mathrm{HO}_{2} \rightarrow \mathrm{H}_{2} \mathrm{O}+\mathrm{O}_{2}$ & $1.0 \mathrm{e} 10$ & & 1 \\
\hline 7 & $\mathrm{OH}+\mathrm{O}_{2}^{-} \rightarrow \mathrm{OH}^{-}+\mathrm{O}_{2}$ & $1.1 \mathrm{e} 10$ & 2120 & 1 \\
\hline 8 & $\mathrm{O}_{3}+\mathrm{OH} \rightarrow \mathrm{HO}_{2}+\mathrm{O}_{2}$ & $1.0 \mathrm{e} 8$ & & 1 \\
\hline $9^{c}$ & $\mathrm{NO}_{3}^{-}+\mathrm{hv} \rightarrow \mathrm{NO}_{2}+\mathrm{OH}+\mathrm{OH}^{-}$ & & & 1 \\
\hline 10 & $\mathrm{~S}(\mathrm{IV})+\mathrm{O}_{2} \stackrel{\mathrm{Fe}^{3+}, \mathrm{Mn}^{2+}}{\longrightarrow} \mathrm{S}(\mathrm{VI})$ & \multicolumn{2}{|c|}{$\begin{array}{l}2.6 \mathrm{e} 3\left[\mathrm{Fe}^{3+}\right]+7.5 \mathrm{e} 2\left[\mathrm{Mn}^{2+}\right] \\
+1.0 \mathrm{e} 10\left[\mathrm{Fe}^{3+}\right]\left[\mathrm{Mn}^{2+}\right] \\
\text { (if } \mathrm{pH} \leq 5.0) \\
7.5 \mathrm{e} 2\left[\mathrm{Mn}^{2+}\right] \\
+2.0 \mathrm{e} 10\left[\mathrm{Fe}^{3+}\right]\left[\mathrm{Mn}^{2+}\right] \\
\text { (if } \mathrm{pH}>5.0 \text { ) }\end{array}$} & 5,6 \\
\hline 11 & $\mathrm{HSO}_{3}^{-}+\mathrm{H}_{2} \mathrm{O}_{2}+\mathrm{H}^{+} \rightarrow \mathrm{SO}_{4}^{2-}+\mathrm{H}_{2} \mathrm{O}+2 \mathrm{H}^{+}$ & $6.9 \mathrm{e} 7$ & 4000 & 1,4 \\
\hline 12 & $\mathrm{HSO}_{3}^{-}+\mathrm{O}_{3} \rightarrow \mathrm{HSO}_{4}^{-}+\mathrm{O}_{2}$ & $3.7 \mathrm{e} 5$ & 5530 & 1,4 \\
\hline 13 & $\mathrm{SO}_{2} \cdot \mathrm{H}_{2} \mathrm{O}+\mathrm{O}_{3} \rightarrow \mathrm{HSO}_{4}^{-}+\mathrm{O}_{2}+\mathrm{H}^{+}$ & $2.4 \mathrm{e} 4$ & & 1,4 \\
\hline 14 & $\mathrm{SO}_{3}^{2-}+\mathrm{O}_{3} \rightarrow \mathrm{SO}_{4}^{2-}+\mathrm{O}_{2}$ & $1.5 \mathrm{e} 9$ & 5280 & 1,4 \\
\hline 15 & $\mathrm{HCHO}+\mathrm{HSO}_{3}^{-} \rightarrow \mathrm{HOCH}_{2} \mathrm{SO}_{3}^{-}$ & $4.5 \mathrm{e} 2$ & 2660 & 4 \\
\hline 16 & $\mathrm{HCHO}+\mathrm{SO}_{3}^{2-} \rightarrow \mathrm{HOCH}_{2} \mathrm{SO}_{3}^{-}+\mathrm{OH}^{-}$ & $5.4 \mathrm{e} 6$ & 2530 & 4 \\
\hline 17 & $\mathrm{HOCH}_{2} \mathrm{SO}_{3}^{-}+\mathrm{OH}^{-} \rightarrow \mathrm{CH}_{2}(\mathrm{OH})_{2}+\mathrm{SO}_{3}^{2-}$ & $4.6 \mathrm{e} 3$ & 4880 & 4 \\
\hline 18 & $\mathrm{HOCH}_{2} \mathrm{SO}_{3}^{-}+\mathrm{OH} \rightarrow \mathrm{HOCHSO}_{3}^{-}+\mathrm{H}_{2} \mathrm{O}$ & 3.0e8 & & 4 \\
\hline 19 & $\mathrm{HOCHSO}_{3}^{-}+\mathrm{O}_{2} \rightarrow \mathrm{HCOOH}+\mathrm{HSO}_{3}^{-}+\mathrm{HO}_{2}$ & $2.5 \mathrm{e} 9$ & & 4 \\
\hline 20 & $\mathrm{CH}_{3} \mathrm{OH}+\mathrm{OH} \rightarrow \mathrm{HCHO}+\mathrm{HO}_{2}$ & $1.0 \mathrm{e} 9$ & 580 & 1 \\
\hline 21 & $\mathrm{CH}_{2}(\mathrm{OH})_{2}+\mathrm{OH} \rightarrow \mathrm{HCOOH}+\mathrm{HO}_{2}+\mathrm{H}_{2} \mathrm{O}$ & $1.1 \mathrm{e} 9$ & 1020 & 1 \\
\hline 22 & $\mathrm{CH}_{3} \mathrm{OO}+\mathrm{HO}_{2} \rightarrow \mathrm{CH}_{3} \mathrm{OOH}+\mathrm{O}_{2}$ & $4.3 \mathrm{e} 5$ & & 4 \\
\hline 23 & $\mathrm{CH}_{3} \mathrm{OO}+\mathrm{O}_{2}^{-} \rightarrow \mathrm{CH}_{3} \mathrm{OOH}+\mathrm{OH}^{-}+\mathrm{O}_{2}$ & $5.0 \mathrm{e} 7$ & & 4 \\
\hline 24 & $\mathrm{CH}_{3} \mathrm{OOH}+\mathrm{OH} \rightarrow \mathrm{CH}_{3} \mathrm{OO}+\mathrm{H}_{2} \mathrm{O}$ & $3.0 \mathrm{e} 7$ & 1680 & 1 \\
\hline 25 & $\mathrm{CH}_{3} \mathrm{OOH}+\mathrm{OH} \rightarrow \mathrm{HO}_{2}+\mathrm{HCOOH}$ & $6.0 \mathrm{e} 6$ & 1680 & 1 \\
\hline 26 & $\mathrm{HCOOH}+\mathrm{OH} \rightarrow \mathrm{CO}_{2}+\mathrm{HO}_{2}+\mathrm{H}_{2} \mathrm{O}$ & $1.3 \mathrm{e} 8$ & 1000 & 1 \\
\hline 27 & $\mathrm{HCOO}^{-}+\mathrm{OH} \rightarrow \mathrm{CO}_{2}+\mathrm{H}_{2} \mathrm{O}$ & $1.0 \mathrm{e} 9$ & 1000 & 1 \\
\hline 28 & $\mathrm{CH}_{3} \mathrm{CHO}+\mathrm{OH} \rightarrow \mathrm{CH}_{3} \mathrm{COOH}+\mathrm{HO}_{2}$ & $3.6 \mathrm{e} 9$ & & 1 \\
\hline 29 & $\mathrm{CH}_{3} \mathrm{CH}(\mathrm{OH})_{2}+\mathrm{OH} \rightarrow \mathrm{CH}_{3} \mathrm{COOH}+\mathrm{HO}_{2}$ & $1.2 \mathrm{e} 9$ & & 1 \\
\hline 30 & $\mathrm{CH}_{3} \mathrm{CH}_{2} \mathrm{OH}+\mathrm{OH} \rightarrow \mathrm{CH}_{3} \mathrm{CHO}+\mathrm{HO}_{2}$ & $1.9 \mathrm{e} 9$ & & 1 \\
\hline 31 & $\mathrm{CH}_{2} \mathrm{OHCHO}+\mathrm{OH} \rightarrow\left(\mathrm{CH}_{2}(\mathrm{OH})_{2}\right)_{2}+\mathrm{HO}_{2}$ & $1.2 \mathrm{e} 9$ & & 2 \\
\hline 32 & $\left(\mathrm{CH}_{2}(\mathrm{OH})_{2}\right)_{2}+\mathrm{OH} \rightarrow \mathrm{CHOCOOH}+\mathrm{HO}_{2}$ & $1.1 \mathrm{e} 9$ & 1516 & 1 \\
\hline 33 & $\mathrm{CHOCOOH}+\mathrm{OH} \rightarrow(\mathrm{COOH})_{2}+\mathrm{HO}_{2}$ & $3.6 \mathrm{e} 8$ & 1000 & 2 \\
\hline 34 & $\mathrm{CHOCOO}^{-}+\mathrm{OH} \rightarrow(\mathrm{COOH})_{2}+\mathrm{HO}_{2}$ & $2.9 \mathrm{e} 9$ & 4300 & 2 \\
\hline 35 & $\mathrm{CH}_{3} \mathrm{COOH}+\mathrm{OH} \rightarrow 0.85 \mathrm{CHOCOOH}+0.15 \mathrm{CH}_{2}(\mathrm{OH})_{2}$ & $1.6 \mathrm{e} 7$ & & 3 \\
\hline 36 & $\mathrm{CH}_{3} \mathrm{COO}^{-}+\mathrm{OH} \rightarrow 0.85 \mathrm{CHOCOOH}+0.15 \mathrm{CH}_{2}(\mathrm{OH})_{2}$ & $8.5 \mathrm{e} 7$ & & 3 \\
\hline 37 & $(\mathrm{COOH})_{2}+\mathrm{OH} \rightarrow \mathrm{HO}_{2}+2 \mathrm{CO}_{2}+\mathrm{H}_{2} \mathrm{O}$ & $1.4 \mathrm{e} 6$ & & 2 \\
\hline 38 & $\mathrm{HC}_{2} \mathrm{O}_{4}^{-}+\mathrm{OH} \rightarrow \mathrm{HO}_{2}+2 \mathrm{CO}_{2}+\mathrm{H}_{2} \mathrm{O}$ & $1.9 \mathrm{e} 8$ & 2800 & 2 \\
\hline 39 & $\mathrm{C}_{2} \mathrm{O}_{4}^{2-}+\mathrm{OH} \rightarrow \mathrm{HO}_{2}+2 \mathrm{CO}_{2}+\mathrm{H}_{2} \mathrm{O}$ & $1.6 \mathrm{e} 8$ & 4300 & 2 \\
\hline 40 & $\mathrm{CH}_{3} \mathrm{COCHO}+\mathrm{OH} \rightarrow \mathrm{CH}_{3} \mathrm{COCOOH}+\mathrm{HO}_{2}$ & $1.1 \mathrm{e} 9$ & 1600 & 2 \\
\hline 41 & $\mathrm{CH}_{3} \mathrm{COCOOH}+\mathrm{OH} \rightarrow \mathrm{CH}_{3} \mathrm{COOH}+\mathrm{HO}_{2}+\mathrm{CO}_{2}$ & $6.0 \mathrm{e} 7$ & & 3 \\
\hline 42 & $\mathrm{CH}_{3} \mathrm{COCOO}^{-}+\mathrm{OH} \rightarrow \mathrm{CH}_{3} \mathrm{COO}^{-}+\mathrm{HO}_{2}+\mathrm{CO}_{2}$ & $6.0 \mathrm{e} 7$ & & 3 \\
\hline $43^{\mathrm{d}}$ & $\mathrm{S} 3+\mathrm{OH} \rightarrow \mathrm{S} 3 \mathrm{PD}+\mathrm{HO}_{2}$ & $4.98 \mathrm{e} 9$ & & 7 \\
\hline $44^{\mathrm{d}}$ & $\mathrm{S} 5+\mathrm{OH} \rightarrow \mathrm{S} 5 \mathrm{PD}+\mathrm{HO}_{2}$ & $5.76 \mathrm{e} 9$ & & 7 \\
\hline $45^{\mathrm{d}}$ & $\mathrm{S} 7+\mathrm{OH} \rightarrow \mathrm{S} 7 \mathrm{PD}+\mathrm{HO}_{2}$ & $4.26 \mathrm{e} 9$ & & 7 \\
\hline $46^{\mathrm{d}}$ & $\mathrm{S}^{-}+\mathrm{OH} \rightarrow \mathrm{S} 7 \mathrm{PD}+\mathrm{HO}_{2}$ & $4.08 \mathrm{e} 9$ & & 7 \\
\hline $47^{\mathrm{d}}$ & $\mathrm{S} 10+\mathrm{OH} \rightarrow \mathrm{S} 10 \mathrm{PD}$ & $1.23 \mathrm{e} 10$ & & 7 \\
\hline
\end{tabular}

a in appropriate units of $\mathrm{M}$ and $\mathrm{s}^{-1}$

b References: 1: Ervens et al. (2003); 2: Ervens et al. (2004); 3: Lim et al. (2005); 4: Warneck (1999); 5. Martin and Good (1991); 6: Zaveri (1999); 7: Dutot et al. (2003).

${ }^{c}$ Maximum photolysis rates for reactions 3 and 9 are $6.01 \mathrm{e}-6 \mathrm{~s}^{-1}$ and $2.41 \mathrm{e}-7 \mathrm{~s}^{-1}$, respectively, in zero-dimensional model simulations. In three-dimensional model simulations, photolysis rate for reaction 3 is calculated by multiplying the corresponding gas-phase photolysis rate by 2.0 , and photolysis rate for reaction 9 is calculated by multiplying photolysis rate for reaction 3 by 0.04 .

d S3: 3-hydroxy-2, 4-dimethyl-2, 4-hexadiendial; S3PD: 3-hydroxy-2, 4-dimethyl-2, 4-hexadienalic acid; S5: 2-hydroxyl-3-isopropyl6-keto-heptanal; S5PD: 2-hydroxyl-3-isopropyl-6-keto-heptanoic acid; S7: 2,4-dimethyl-3-formyl-benzoic acid; S7PD: 3, 5-dimethyl-1, 4-dibenzoic acid; S10: 1-methyl-1-hydroxy-2-nitrato-4-isopropyl- cyclohexane; S10PD: 1-methyl-1, 3-dihydroxy-2-nitrato-4-isopropylcyclohexane 
Table 2. Aqueous-phase equilibrium reactions included in AqChem.

\begin{tabular}{|c|c|c|c|c|}
\hline Num. & Equilibrium reactions & $\mathrm{K}(\mathrm{M})$ & $\mathrm{E} / \mathrm{R}(\mathrm{K})$ & Ref. $^{\mathrm{a}}$ \\
\hline 1 & $\mathrm{H}_{2} \mathrm{O} \leftrightarrow \mathrm{H}^{+}+\mathrm{OH}^{-}$ & $1.8 \mathrm{e}-16$ & 6800 & 1 \\
\hline 2 & $\mathrm{CO}_{2} \cdot \mathrm{H}_{2} \mathrm{O} \leftrightarrow \mathrm{HCO}_{3}^{+}+\mathrm{H}^{-}$ & $7.7 \mathrm{e}-7$ & -1000 & 2 \\
\hline 3 & $\mathrm{HCO}_{3}^{+} \leftrightarrow \mathrm{CO}_{3}^{2-}+\mathrm{H}^{-}$ & $4.84 \mathrm{e}-11$ & -1760 & 2 \\
\hline 4 & $\mathrm{HO}_{2} \leftrightarrow \mathrm{H}^{+}+\mathrm{O}_{2}^{-}$ & $1.6 e-5$ & & 1 \\
\hline 5 & $\mathrm{HNO}_{4} \leftrightarrow \mathrm{H}^{+}+\mathrm{NO}_{4}^{-}$ & $1.0 \mathrm{e}-5$ & & 1 \\
\hline 6 & $\mathrm{HNO}_{3} \leftrightarrow \mathrm{H}^{+}+\mathrm{NO}_{3}^{-}$ & 22.0 & -1800 & 1 \\
\hline 7 & $\mathrm{HONO} \leftrightarrow \mathrm{H}^{+}+\mathrm{NO}_{2}^{-\frac{3}{-}}$ & $5.3 e-4$ & 1760 & 1 \\
\hline 8 & $\mathrm{NH}_{3} \cdot \mathrm{H}_{2} \mathrm{O} \leftrightarrow \mathrm{NH}_{4}{ }^{2}+\mathrm{OH}^{-}$ & $1.75 \mathrm{e}-5$ & 560 & 1 \\
\hline 9 & $\mathrm{SO}_{2} \cdot \mathrm{H}_{2} \mathrm{O} \leftrightarrow \mathrm{HSO}_{3}^{-}+\mathrm{H}^{+}$ & $1.73 \mathrm{e}-2$ & -1940 & 1 \\
\hline 10 & $\mathrm{HSO}_{3}^{-} \leftrightarrow \mathrm{SO}_{3}^{2-}+\mathrm{H}^{+}$ & $6.22 \mathrm{e}-8$ & -1960 & 1 \\
\hline 11 & $\mathrm{H}_{2} \mathrm{SO}_{4} \leftrightarrow \mathrm{HSO}_{4}^{-}+\mathrm{H}^{+}$ & 1000 & & 1 \\
\hline 12 & $\mathrm{HSO}_{4}^{-} \leftrightarrow \mathrm{SO}_{4}^{2-}+\mathrm{H}^{+}$ & $1.02 \mathrm{e}-2$ & -2700 & 1 \\
\hline 13 & $\mathrm{HCHO}+\mathrm{H}_{2} \mathrm{O} \leftrightarrow \mathrm{CH}_{2}(\mathrm{OH})_{2}$ & 36.0 & -4030 & 1 \\
\hline 14 & $\mathrm{HCOOH} \leftrightarrow \mathrm{HCOO}^{-}+\mathrm{H}^{+}$ & $1.77 \mathrm{e}-4$ & -12 & 1 \\
\hline 15 & $\mathrm{CH}_{3} \mathrm{CHO}+\mathrm{H}_{2} \mathrm{O} \leftrightarrow \mathrm{CH}_{3} \mathrm{CH}(\mathrm{OH})_{2}$ & $2.46 \mathrm{e}-2$ & -2500 & 1 \\
\hline 16 & $\mathrm{CH}_{3} \mathrm{COOH} \leftrightarrow \mathrm{H}^{+}+\mathrm{CH}_{3} \mathrm{COO}^{-}$ & $1.75 \mathrm{e}-5$ & -46 & 1 \\
\hline 17 & $\mathrm{CHOCOOH} \leftrightarrow \mathrm{CHOCOO}^{-}+\mathrm{H}^{+}$ & $6.6 \mathrm{e}-4$ & & 2 \\
\hline 18 & $\mathrm{COOHCOOH} \leftrightarrow \mathrm{HC}_{2} \mathrm{O}_{4}^{-}+\mathrm{H}^{+}$ & $6.4 \mathrm{e}-2$ & & 1 \\
\hline 19 & $\mathrm{HC}_{2} \mathrm{O}_{4}^{-} \leftrightarrow \mathrm{C}_{2} \mathrm{O}_{4}^{2-}+\mathrm{H}^{+}$ & $5.25 \mathrm{e}-5$ & & 1 \\
\hline 20 & $\mathrm{CH}_{3} \mathrm{COCOOH} \stackrel{4}{\leftrightarrow} \mathrm{CH}_{3} \mathrm{COCOO}^{-}+\mathrm{H}^{+}$ & $4.07 e-3$ & & 2 \\
\hline 21 & $\mathrm{~S} 7 \leftrightarrow \mathrm{S}^{-}+\mathrm{H}^{+}$ & $7.335 \mathrm{e}-5$ & & 3 \\
\hline $22^{\mathrm{b}}$ & $\mathrm{S} 3 \mathrm{PD} \leftrightarrow \mathrm{S} \mathrm{PD}^{-}+\mathrm{H}^{+}$ & $3.7 \mathrm{e}-5$ & & 3 \\
\hline $23^{\mathrm{b}}$ & $\mathrm{S} 5 \mathrm{PD} \leftrightarrow \mathrm{S} \mathrm{PD}^{-}+\mathrm{H}^{+}$ & $6.52 \mathrm{e}-4$ & & 3 \\
\hline $24^{\mathrm{b}}$ & 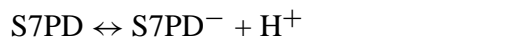 & $1.7 \mathrm{e}-3$ & & 3 \\
\hline
\end{tabular}

a References: 1: Ervens et al. (2003); 2: Ervens et al. (2004); 3: Pun et al. (2002).

${ }^{\mathrm{b}}$ See Table 1 for molecular definition of S7, S3PD, S5PD, and S7PD.

al., 2006). If clouds are present, AqChem is first called to simulate the cloud processing of organics, followed by a call to MPMPO to calculate SOA formation. The organic oxidation products in clouds were lumped into existing surrogates in the MPMPO module. Oxalic and pyruvic acids were lumped into surrogate 1 (oxalic acid) of MPMPO. S3PD, S5PD, S7PD, and S10PD were lumped into surrogate 2 (S2, 2-methyl-5-formyl-2, 4-hexadiendioic acid), surrogate 4 (S4, 2-hydroxy-3-isopropyl-5-keto-3-hexenoic acid), surrogate 6 (S6, 3, 5-dimethyl-2-nitro-4-hydroxy-benzoic acid), and surrogate 5 (S5, 2-hydroxy-3-isopropyl-6-keto-heptanal) of MPMPO, respectively. Overall, the aqueous products S3PD, S5PD, S7PD, and S10PD are less volatile than their precursors, as in the treatment used by Gelencser and Varga (2005). Vapor pressure and $K_{H}$ estimates for the surrogate species in MPMPO can be found in Pun et al. (2002) and Griffin et al. (2005).

The kinetic processor KPP2.1 (Damian et al., 2002; Daescu et al., 2003; Sandu et al., 2003) was used to process AqChem and generate the associated numerical codes. A Rosenbrock solver in KPP2.1 was used to solve the differential equations associated with the mechanism (Sandu et al., 1997).

\subsection{Zero-dimensional model simulations}

A zero-dimensional model was constructed to investigate potential SOA formation due to irreversible aqueous-phase organic chemistry under scenarios for which clouds are present for three consecutive hours per day (Lim et al., 2005). The zero-dimensional model considers emissions, dry deposition, and gas- and aqueous-phase chemistry for chemical species. The mass balance of the gas- and aqueous-phase species is described by (Pandis and Seinfeld, 1989; Lim et al., 2005)

$$
\begin{aligned}
& \frac{d C_{g}}{d t}=Q_{g}-S_{g}+\frac{E}{Z}-\frac{v_{d} C_{g}}{Z}-L k C_{g}+\frac{L k C_{a}}{K_{H} R T} \\
& \frac{d C_{a}}{d t}=Q_{a}-S_{a}+k C_{g}-\frac{k C_{a}}{K_{H} R T}
\end{aligned}
$$

where $C_{g}$ is the gas-phase concentration (mole $\mathrm{L}^{-1}$ of air), $C_{a}$ is the aqueous-phase concentration (M), $Q_{g}$ (mole $^{-1}$ of air s $\left.{ }^{-1}\right)$ and $Q_{a}\left(\mathrm{M} \mathrm{sec}^{-1}\right)$ are the gas-phase and aqueousphase production rate, respectively, $S_{g}\left(\right.$ mole $\mathrm{L}^{-1}$ of air s ${ }^{-1}$ ) and $S_{a}\left(\mathrm{M} \mathrm{s}^{-1}\right)$ are the gas-phase and aqueous-phase loss rate, respectively, $E$ (mole $\mathrm{dm}^{-2} \mathrm{~s}^{-1}$ ) is the emission rate of the gas-phase species, $Z(\mathrm{dm})$ is the boundary layer height, 
Table 3. Uptake parameters for gas-phase species in AqChem.

\begin{tabular}{|c|c|c|c|c|c|}
\hline Species $^{\mathrm{a}}$ & $\mathrm{K}_{H, 298 \mathrm{~K}}\left(\mathrm{M} \mathrm{atm}^{-1}\right)$ & $\Delta \mathrm{H} / \mathrm{R}(\mathrm{K})$ & $\alpha$ & $\mathrm{D}_{g}\left(\mathrm{~m}^{2} \mathrm{~s}^{-1}\right)$ & Ref. $^{b}$ \\
\hline $\mathrm{CO}_{2}$ & $3.11 \mathrm{e}-2$ & -2423 & $2.0 \mathrm{e}-4$ & $1.55 \mathrm{e}-5$ & 1 \\
\hline $\mathrm{NH}_{3}$ & 60.7 & -3920 & 0.04 & $2.3 e-5$ & 1 \\
\hline $\mathrm{O}_{3}$ & $1.14 \mathrm{e}-2$ & -2300 & 0.05 & $1.48 \mathrm{e}-5$ & 1 \\
\hline $\mathrm{HO}_{2}$ & $9.0 \mathrm{e} 3$ & & 0.01 & $1.04-5$ & 1 \\
\hline $\mathrm{OH}$ & 25.0 & -5280 & 0.05 & $1.53 e-5$ & 1 \\
\hline $\mathrm{H}_{2} \mathrm{O}_{2}$ & $1.02 \mathrm{e} 5$ & -6340 & 0.11 & $1.46 \mathrm{e}-5$ & 1 \\
\hline $\mathrm{HNO}_{3}$ & $2.1 \mathrm{e} 5$ & -8700 & 0.054 & $1.32 \mathrm{e}-5$ & 1 \\
\hline HONO & 49.0 & -4880 & 0.5 & $1.30 \mathrm{e}-5$ & 1 \\
\hline $\mathrm{HNO}_{4}$ & $3.0 \mathrm{e} 4$ & & 0.1 & $1.30 \mathrm{e}-5$ & 1 \\
\hline $\mathrm{SO}_{2}$ & 1.24 & -3247 & $3.5 \mathrm{e}-2$ & $1.28 \mathrm{e}-5$ & 1 \\
\hline $\mathrm{H}_{2} \mathrm{SO}_{4}$ & $2.1 \mathrm{e} 5$ & & 0.07 & $1.30 \mathrm{e}-5$ & 1 \\
\hline $\mathrm{HCHO}$ & 2.5 & & 0.02 & $1.64 \mathrm{e}-5$ & 1 \\
\hline $\mathrm{CH}_{3} \mathrm{OH}$ & 220 & -5390 & 0.015 & $1.16 \mathrm{e}-5$ & 1 \\
\hline $\mathrm{CH}_{3} \mathrm{OO}$ & 310 & & $3.8 \mathrm{e}-3$ & $1.35 e-5$ & 1 \\
\hline $\mathrm{CH}_{3} \mathrm{OOH}$ & 310 & & $3.8 \mathrm{e}-3$ & $1.31 \mathrm{e}-5$ & 1 \\
\hline $\mathrm{HCOOH}$ & 5530 & 5630 & 0.10 & $1.0 \mathrm{e}-5$ & 2 \\
\hline $\mathrm{CH}_{3} \mathrm{CHO}$ & 11.4 & -6254 & 0.03 & $1.22 \mathrm{e}-5$ & 1 \\
\hline $\mathrm{CH}_{3} \mathrm{CH}_{2} \mathrm{OH}$ & 190 & -6290 & $8.2 \mathrm{e}-3$ & $0.95 e-5$ & 1 \\
\hline $\mathrm{CH}_{2} \mathrm{OHCHO}$ & $4.14 \mathrm{e} 4$ & & 0.03 & $1.95 e-5$ & 2 \\
\hline $\mathrm{CH}_{3} \mathrm{COOH}$ & 5500 & -5890 & 0.019 & $1.24 \mathrm{e}-5$ & 2 \\
\hline $\mathrm{COOHCOOH}$ & $3.26 \mathrm{e} 6$ & & 0.019 & $1.24 \mathrm{e}-5$ & 3 \\
\hline CHOCHO & $3.0 \mathrm{e} 5$ & & $3.8 \mathrm{e}-3$ & $1.31 \mathrm{e}-5$ & 2 \\
\hline $\mathrm{CHOCOOH}$ & 9000 & & 0.1 & $1.0 \mathrm{e}-5$ & 2 \\
\hline $\mathrm{CH}_{3} \mathrm{COCHO}$ & 3710 & & 0.023 & $1.15 \mathrm{e}-5$ & 2 \\
\hline $\mathrm{CH}_{3} \mathrm{COCOOH}$ & $3.11 \mathrm{e} 5$ & 5100 & 0.1 & $1.0 \mathrm{e}-5$ & 2 \\
\hline$S 3^{\mathrm{c}}$ & $6.01 \mathrm{e} 6$ & & 0.05 & $1.0 \mathrm{e}-5$ & 4,5 \\
\hline $\mathrm{S} 5^{\mathrm{c}}$ & $3.90 \mathrm{e} 7$ & & 0.05 & $1.0 \mathrm{e}-5$ & 4,5 \\
\hline$S 7^{\mathrm{c}}$ & $4.91 \mathrm{e} 6$ & & 0.05 & $1.0 \mathrm{e}-5$ & 4,5 \\
\hline $\mathrm{S} 10^{\mathrm{c}}$ & $1.18 \mathrm{e} 6$ & & 0.05 & $1.0 \mathrm{e}-5$ & 4,5 \\
\hline
\end{tabular}

a $\mathrm{N}_{2} \mathrm{O}_{5}$ is assumed to be completely dissolved in water and to form two moles of $\mathrm{HNO}_{3}$.

b References: 1: Ervens et al. (2003); 2: Ervens et al. (2004); 3: Lim et al. (2005); 4: Pun et al. (2002); 5: Aumont et al. (2000).

c See Table 1 for molecular definition of S3, S5, S7, and S10.

$v_{d}\left(\mathrm{dm} \mathrm{s}^{-1}\right)$ is the dry deposition velocity, $L$ is the cloud liquid water volume fraction, $K_{H}\left(\mathrm{M} \mathrm{atm}^{-1}\right)$ is the effective Henry's law constant, $R\left(\mathrm{~L} \mathrm{~atm} \mathrm{~mole}{ }^{-1} \mathrm{~K}^{-1}\right)$ is the ideal gas constant, $T(\mathrm{~K})$ is the temperature, and $k\left(\mathrm{~s}^{-1}\right)$ is the mass transfer coefficient,

$k=\left(\frac{a^{2}}{3 D_{g}}+\frac{4 a}{3 v \alpha}\right)^{-1}$

where $\alpha$ is the mass accommodation coefficient, $D_{g}$ $\left(\mathrm{cm}^{2} \mathrm{~s}^{-1}\right)$ is the gas-phase diffusion constant, $a$ is the cloud droplet radius $(\mathrm{cm})$, and $v$ is the mean molecular speed $\left(\mathrm{cm} \mathrm{s}^{-1}\right)$.

The zero-dimensional model couples CACM, AqChem, and MPMPO. Emissions and dry deposition of gas-phase species were implemented within the gas-phase chemistry mechanism. As with AqChem, the latest version of CACM with emissions and dry deposition of species was processed by KPP2.1, and a Rosenbrock solver was used for solving the differential equations. If clouds are present, the simulations of gas-phase and aqueous-phase chemistry processes are split every $12 \mathrm{~min}$, following the treatment in the threedimensional model that will be discussed subsequently. If clouds are not present, only gas-phase chemistry is simulated every $12 \mathrm{~min}$. At the end of every $12 \mathrm{~min}$, MPMPO is called to simulate SOA formation.

For the zero-dimensional model simulation, conditions are based on the study of Lim et al. (2005). The boundary layer height was assumed to be fixed at $1000 \mathrm{~m}$. Temperature and relative humidity (RH) are $298 \mathrm{~K}$ and $75 \%$, respectively, if clouds are not present. Clouds were assumed to be present through the entire boundary layer for hours 13-16 of each day. Cloud water content was assumed to be $0.5 \mathrm{~g} \mathrm{~m}^{-3}$, and cloud droplets were assumed to have a uniform diameter of $10 \mu \mathrm{m}$ (Gelencser and Varga, 2005; Lim et al., 2005). Temperature and RH were assumed to be $288 \mathrm{~K}$ and $100 \%$, respectively, when clouds are present. In addition, a constant 
Table 4. Twenty-four-hour average emission rates $\left(\right.$ moles $\mathrm{m}^{-2} \mathrm{~s}^{-1}$ ) for two scenarios used in zero-dimensional model simulations.

\begin{tabular}{|c|c|c|}
\hline Species & $\begin{array}{l}\text { Emissions for } \\
\text { Scenario } 1\end{array}$ & $\begin{array}{l}\text { Emissions for } \\
\text { Scenario } 2\end{array}$ \\
\hline NO & $1.36 \mathrm{e}-9$ & $3.27 \mathrm{e}-8$ \\
\hline $\mathrm{NO}_{2}$ & $1.42 \mathrm{e}-10$ & $3.63 \mathrm{e}-9$ \\
\hline $\mathrm{CO}$ & $1.33 \mathrm{e}-8$ & $3.40 \mathrm{e}-7$ \\
\hline $\mathrm{HCHO}$ & $1.33 \mathrm{e}-11$ & $6.73 \mathrm{e}-10$ \\
\hline ALD1 ${ }^{a}$ & $2.63 \mathrm{e}-10$ & $1.06 \mathrm{e}-9$ \\
\hline OLEL $^{\mathrm{a}}$ & $1.38 \mathrm{e}-9$ & $1.49 \mathrm{e}-9$ \\
\hline $\mathrm{AROL}^{\mathrm{a}}$ & $5.56 \mathrm{e}-11$ & $7.18 \mathrm{e}-10$ \\
\hline $\mathrm{AROH}^{\mathrm{a}}$ & $6.60 \mathrm{e}-11$ & $1.33 \mathrm{e}-9$ \\
\hline ISOPa & $2.64 \mathrm{e}-9$ & $1.83 e-9$ \\
\hline TERPa & $8.30 \mathrm{e}-10$ & $1.87 \mathrm{e}-11$ \\
\hline $\mathrm{ALKL}^{\mathrm{a}}$ & $3.04 \mathrm{e}-9$ & $1.11 \mathrm{e}-8$ \\
\hline $\mathrm{SO}_{2}$ & $5.06 \mathrm{e}-11$ & $6.38 \mathrm{e}-9$ \\
\hline
\end{tabular}

a ALD1: lumped small-carbon-number aldehydes; OLEL: lumped alkenes $\mathrm{C}_{3}-\mathrm{C}_{6}$; AROL: lumped low-SOA-yield aromatic species; AROH: lumped high-SOA-yield aromatic species; ISOP: isoprene; TERP: monoterpenes; ALKL: lumped alkanes $\mathrm{C}_{2}-\mathrm{C}_{6}$.

cloud $\mathrm{pH}$ of 4.5 was assumed. Photolysis rates were assumed to vary semisinusoidally between hour 6 and hour 18 of each day, with a peak at hour 12 . In addition, photolysis rates were assumed not to be influenced by the presence of clouds, as was assumed by Ervens et al. (2004) and Lim et al. (2005).

Two emissions scenarios were studied. Scenario 1 features strong biogenic monoterpene emissions for a rural area, and Scenario 2 features strong anthropogenic emissions of aromatic compounds for an urban area. Emission rates and deposition velocities of gas-phase hydrocarbons were extracted from previous three-dimensional model simulations (Chen et al., 2006; Mao et al., 2006). These are shown in Tables 4 and 5 , respectively. In order to simulate SOA formation in MPMPO, total POA concentrations (fractions of individual POA species are consistent between the two scenarios and are based on those used in Chen et al., 2006), aerosol water content, and aerosol $\mathrm{pH}$ for both scenarios were assumed to be constant at $1.0 \mu \mathrm{g} \mathrm{m}^{-3}, 30 \mu \mathrm{g} \mathrm{m}^{-3}$, and 3.0, respectively. This simplified treatment omits considerations of emission, deposition, and size distribution of aerosols. In addition, the zero-dimensional model simulations were performed for four days for each scenario. The first two days were used as initialization days, while the last two days were used for analysis.

\subsection{Three-dimensional model simulation}

AqChem was incorporated into the Community Multiscale Air Quality (CMAQ) model version 4.4 with CACM and MPMPO (Chen et al., 2006). In addition, the computationally efficient version of the MPMPO module (Tulet et al.,
Table 5. Twenty-four-hour average dry deposition velocities $\left(\mathrm{dm} \mathrm{s}^{-1}\right)$ for two scenarios used in zero-dimensional model simulations.

\begin{tabular}{|c|c|c|}
\hline Species & $\begin{array}{l}\text { Dry deposition velocities } \\
\text { for Scenario } 1\end{array}$ & $\begin{array}{l}\text { Dry deposition velocities } \\
\text { for Scenario } 2\end{array}$ \\
\hline $\mathrm{NO}$ & $2.52 \mathrm{e}-3$ & $9.08 \mathrm{e}-4$ \\
\hline $\mathrm{NO}_{2}$ & $2.03 \mathrm{e}-3$ & $7.45 \mathrm{e}-4$ \\
\hline $\mathrm{O}_{3}$ & $2.71 \mathrm{e}-3$ & $1.46 \mathrm{e}-3$ \\
\hline $\mathrm{H}_{2} \mathrm{O}_{2}$ & $7.41 \mathrm{e}-3$ & $4.77 \mathrm{e}-3$ \\
\hline $\mathrm{NO}_{3}$ & $1.48 \mathrm{e}-2$ & $3.26 \mathrm{e}-2$ \\
\hline $\mathrm{HNO}_{3}$ & $1.75 \mathrm{e}-2$ & $3.41 \mathrm{e}-2$ \\
\hline HONO & $6.88 \mathrm{e}-3$ & $3.94 \mathrm{e}-3$ \\
\hline $\mathrm{N}_{2} \mathrm{O}_{5}$ & $1.56 \mathrm{e}-2$ & $3.00 \mathrm{e}-2$ \\
\hline $\mathrm{CO}$ & $2.83 \mathrm{e}-3$ & $1.26 \mathrm{e}-3$ \\
\hline $\mathrm{SO}_{2}$ & $6.28 \mathrm{e}-3$ & $3.82 \mathrm{e}-3$ \\
\hline $\mathrm{HCHO}$ & $6.67 \mathrm{e}-3$ & $3.20 \mathrm{e}-3$ \\
\hline $\operatorname{ALD}^{a}$ & $4.03 e-3$ & $2.03 \mathrm{e}-3$ \\
\hline $\mathrm{ORA}^{\mathrm{a}}$ & $6.06 \mathrm{e}-3$ & $3.63 e-3$ \\
\hline $\mathrm{PAN}^{\mathrm{a}}$ & $1.95 \mathrm{e}-3$ & $8.89 \mathrm{e}-4$ \\
\hline
\end{tabular}

a ALD for general aldehydes, ORA for general organic acids, and PAN for general peroxy acyl nitrates.

2006) was used. CMAQ originally employed the RADM aqueous-phase chemistry mechanism, mainly for the purpose of predicting aqueous-phase sulfate formation in grid resolved clouds and/or sub-grid convective clouds (Byun and Ching, 1999). In the CMAQ model, the aqueous-phase chemistry mechanism is called when the liquid water content of clouds exceeds $10^{-5} \mathrm{~kg} \mathrm{~m}^{-3}$. Cloud droplet diameter was assumed to be $10 \mu \mathrm{m}$ (Lim et al., 2005). In addition, CMAQ assumes that accumulation mode particles serve as cloud condensation nuclei and Aitken mode particles form interstitial aerosol that can be scavenged by clouds. Therefore, all accumulation mode particles and some fraction of Aitken mode particles are included in the resulting aqueous phase of the hydrometeors. Chemical species (e.g., nitrate, oxalic acid, S3, S5, S7, and S10) of cloud-incorporated particles are also subject to aqueous-phase chemistry. SOA formation due to both the gas-phase oxidation and the aqueous-phase oxidation is distributed between accumulation and Aitken mode particles using the proportion of preexisting $\mathrm{OA}$ in these two modes.

CMAQ with CACM and MPMPO was applied previously to an episode (3-4 August 2004 with spin-up days of 1-2 August 2004) over the eastern United States (Chen et al., 2006). In this study, CMAQ with CACM and MPMPO and with the newly developed AqChem was applied to the same episode to study the significance of SOA formation from the consideration of irreversible aqueous-phase organic chemistry in clouds. Model inputs (e.g., emissions and meteorological fields) and model configurations (e.g., model domain 


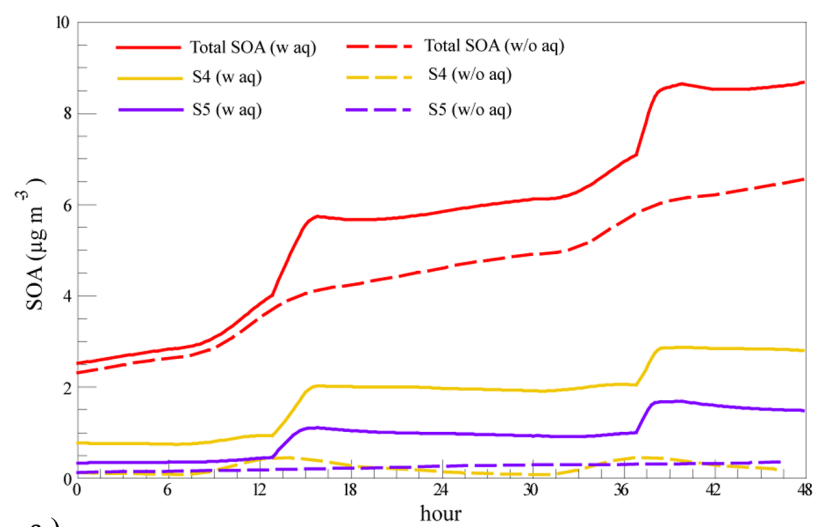

a.)

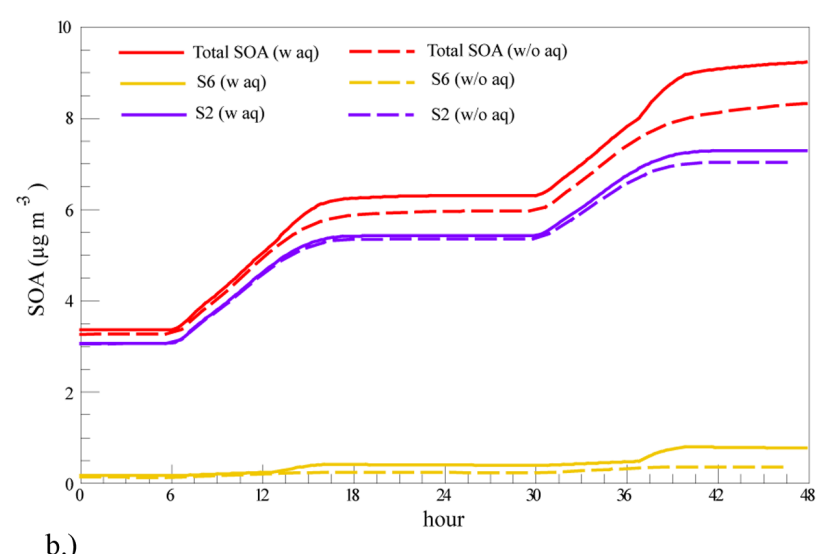

b.)

Fig. 3. SOA predictions from zero-dimensional model simulations: (a) Scenario 1 (S4: SOA of surrogate species 4 of MPMPO; S5: SOA of surrogate species 5 of MPMPO) (b) Scenario 2 (S2: SOA of surrogate species 2 of MPMPO; S6: SOA of surrogate species 6 of MPMPO). "w aq" indicates simulation with consideration of the aqueous-phase organic chemistry in clouds; "w/o aq" indicates simulation without consideration of the aqueous-phase organic chemistry in clouds.

and vertical layers) for this episode are described in Mao et al. (2006) and Chen et al. (2006).

\section{Results}

\subsection{Zero-dimensional model simulations}

Figure 3a shows the SOA predictions from the zerodimensional model simulation for Scenario 1, which features strong monoterpene emissions for a rural area. Averaged over $48 \mathrm{~h}$, total SOA prediction considering SOA formation from cloud-phase organic reactions is $5.7 \mu \mathrm{g} \mathrm{m}^{-3}$, which is approximately $27 \%$ higher than the prediction without considering aqueous-phase chemistry in clouds. At the end of simulation, total SOA prediction with aqueous-phase chemistry in clouds is $8.7 \mu \mathrm{g} \mathrm{m}^{-3}$, which is $32 \%$ more than that from the simulation without clouds. Compared to the SOA prediction without consideration of the aqueous-phase chemistry, total SOA prediction with aqueous-phase chemistry increases substantially during the hours 13-16 and hours 3740 , during which clouds are present. This suggests substantial rapid processing of organic compounds in cloud droplets and that such processing leads to formation of SOA.

As shown in Fig. 3a, the increase of SOA prediction from aqueous-phase chemistry is due primarily to the increase of SOA formation from S4 and S5 of MPMPO, which is attributed to the oxidation of S5 to form S5PD (lumped to S4) and the oxidation of S10 to form S10PD (lumped to S5) in AqChem, respectively. Because S4 and S5 are less volatile and more soluble than S5 and S10, respectively, more SOA is formed. Both S5 and S10 are surrogates for oxidation products of monoterpenes.

Figure $3 \mathrm{~b}$ shows the SOA production from Scenario 2 using the same zero-dimensional model. Scenario 2 represents an urban environment with strong anthropogenic emissions (e.g., nitrogen oxides $\left(\mathrm{NO}_{\mathrm{x}}\right)$ and aromatic compounds). If aqueous-phase chemistry in clouds is considered, the SOA concentrations predicted at the end of the simulation period and averaged over $48 \mathrm{~h}$ are $9.2 \mu \mathrm{g} \mathrm{m}^{-3}$ and $6.3 \mu \mathrm{g} \mathrm{m}^{-3}$, respectively, which are $11 \%$ and $7 \%$ higher than that from simulation without consideration of cloud chemistry. Individually, the increase of SOA prediction by considering aqueousphase organic chemistry is due to the increase of SOA contributions from S2, S6, and, to a smaller extent, $\mathrm{S} 1$, which is then attributed to the aqueous-phase conversion of S3, S7, and organic compounds of carbon number less than four. S2, S6, and the majority of S1 are surrogates for oxidation products from aromatic compounds. Based on the aqueousphase organic chemistry proposed in this study, the effect of aqueous-phase chemistry in clouds on SOA formation is less important for aromatic compounds than it is for monoterpene species. The reasons for this include smaller $\mathrm{OH}$ concentrations in Scenario 2, smaller predicted aromatic product concentrations (from the gas-phase chemistry mechanism) that are subject to the aqueous-phase processing, and smaller reactivity with $\mathrm{OH}$ in cloud droplets for aromatic products compared to monoterpene products.

Sensitivity analyses also were performed to study the effects of RH, temperature in clouds, cloud water content, and reduced photolysis rates in clouds as compared to clear sky conditions on the zero-dimensional model results. It was found that variations in cloud water content have the most significant effect on the model results, with variations in other factors only having minor effects. When cloud water content is reduced from $0.5 \mathrm{~g} \mathrm{~m}^{-3}$ to $0.1 \mathrm{~g} \mathrm{~m}^{-3}$, the increase of SOA prediction considering aqueous-phase organic reactions averaged over 48 hours decreases to $11 \%$ for Scenario 1 and $5 \%$ for Scenario 2 . This suggests clearly that the magnitude of cloud water content needs to be taken into account when results from various model simulations are compared. 

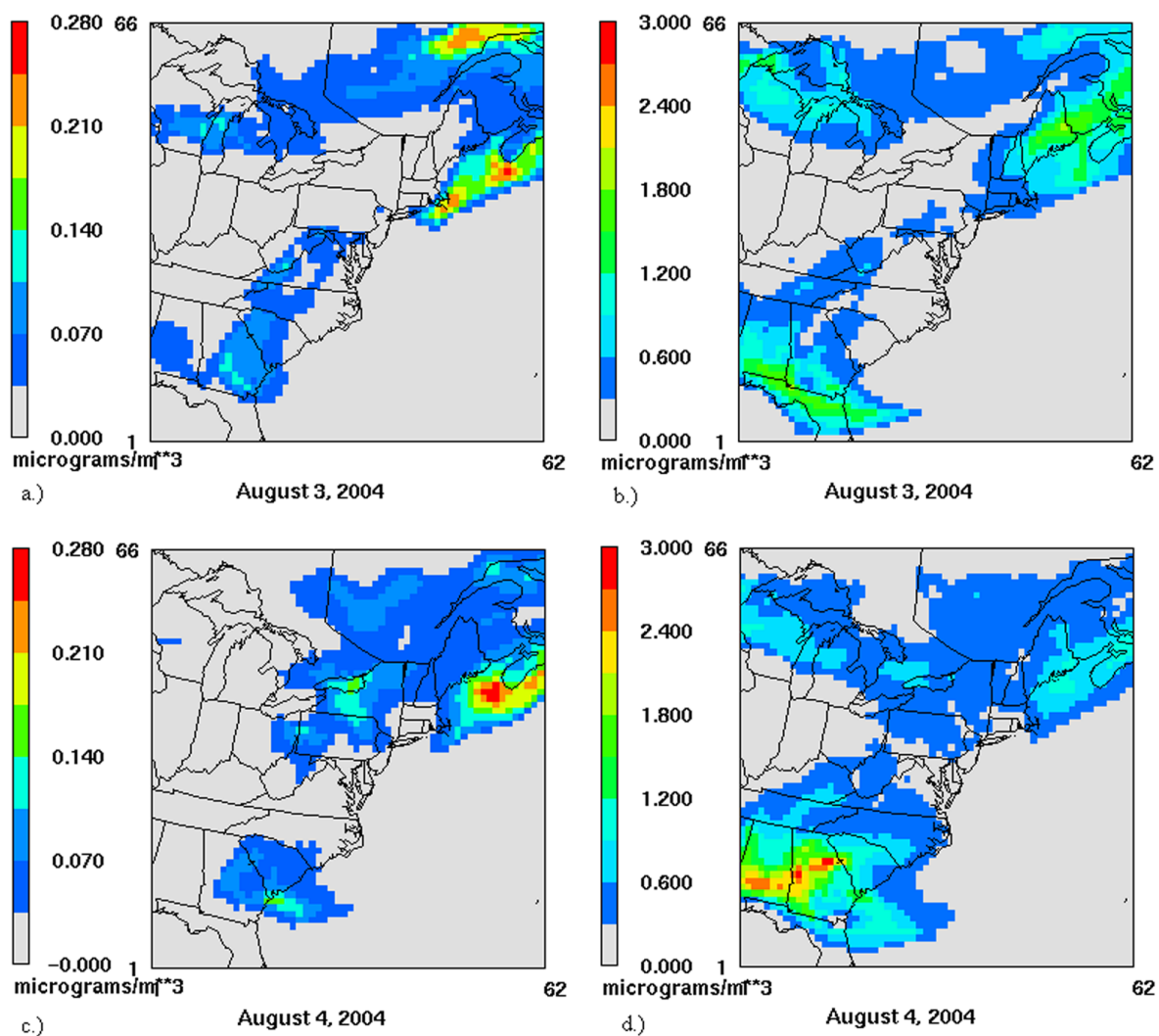

b.)

August 3, 2004

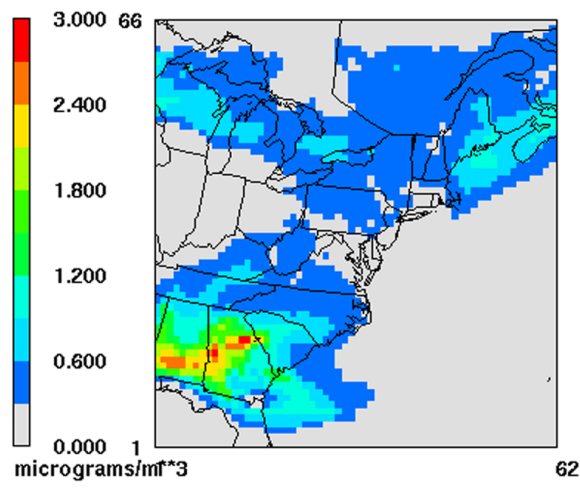

d.)

August 4, 2004

Fig. 4. Spatial distribution of surface SOA predictions: (a) difference between 24-h average SOA prediction on 3 August 2004, with/without aqueous-phase organic chemistry simulation in clouds; (b) 24-h average SOA prediction on 3 August 2004, with aqueous-phase organic chemistry simulation in clouds; (c) difference between 24-h average SOA prediction on 4 August 2004, with/without aqueous-phase organic chemistry simulation in clouds; (d) 24-h average SOA prediction on 4 August 2004, with aqueous-phase organic chemistry simulation in clouds.

\subsection{Three-dimensional model simulation}

Figure 4 shows the difference between 24-h average SOA predictions at the surface for 3-4 August 2004 (UTC) when the SOA formation from aqueous-phase organic chemistry in cloud droplets is considered and not. In addition, the absolute values of SOA predictions at the surface layer for these two days are also shown if the SOA formation from aqueousphase organic chemistry in clouds is considered. Consideration of SOA formation from aqueous-phase organic chemistry increases 24 -h average SOA predictions. The maximum difference in 24-h average SOA predictions within the domain is $0.28 \mu \mathrm{g} / \mathrm{m}^{3}$ for both days. Averaged over the entire domain, the relative increases of 24-h average SOA predictions with consideration of SOA formation from aqueousphase organic chemistry in clouds are $8.7 \%$ and $8.5 \%$ for 3 August and 4 August 2004, respectively.

The increase of SOA predictions if aqueous-phase organic chemistry is considered is due mainly to the cloud processing of S5 and S10, which leads to the formation of less volatile products. Both S5 and S10 are lumped species for oxidation products of monoterpenes. This is consistent with the fact that the majority of SOA predicted within the domain is from biogenic monoterpenes (Chen et al., 2006).

The average relative increase across the domain is smaller than that from the zero-dimensional model simulation for Scenario 1 in which strong monoterpene emissions are considered. The zero-dimensional model simulation represents an ideal situation in which a site is influenced by biogenic monoterpene emissions and is exposed to clouds for three hours in the afternoon (12.5\% of the time of each day). Examination of the meteorological inputs for the chosen episode indicates that resolved clouds occupy approximately $10-20 \%$ of the surface area during the episode. In addition, CMAQ internally calculates sub-grid convective clouds. Therefore, on average, each grid has the probability of 10 $20 \%$ of being exposed to cloud processing, which is close to the cloud exposure time used in the zero-dimensional model. However, the average cloud liquid water content during the episode is about $0.2 \mathrm{~g} \mathrm{~m}^{-3}$, which is smaller than the $0.5 \mathrm{~g} \mathrm{~m}^{-3}$ used in the zero-dimensional model simulation. If cloud liquid water content in the zero-dimensional simulation is assumed to be $0.2 \mathrm{~g} \mathrm{~m}^{-3}$, the SOA prediction averaged over 48 hours considering aqueous-phase organic 


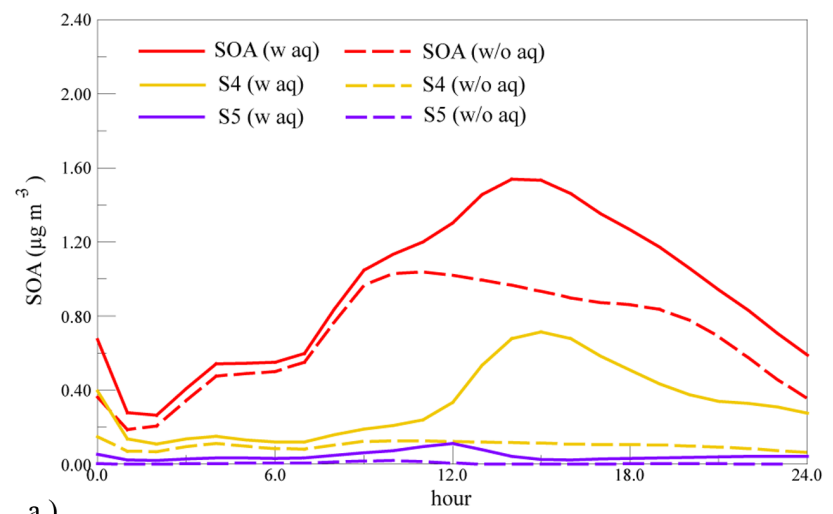

a.)

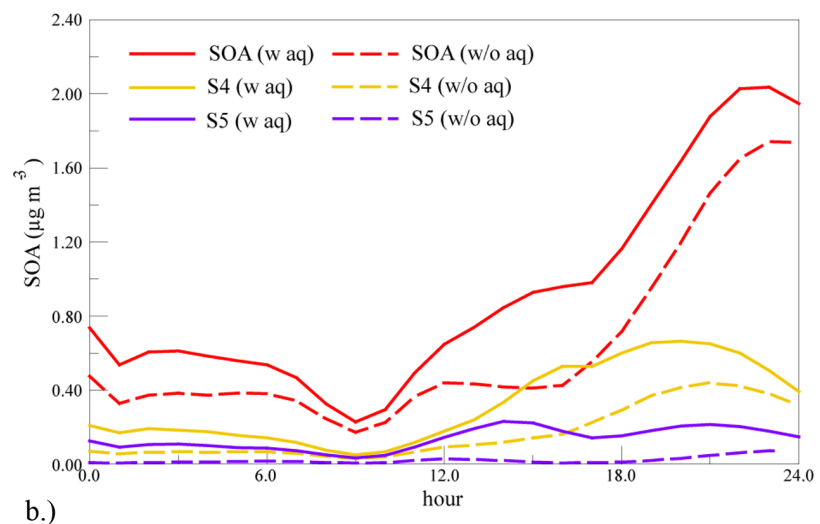

Fig. 5. Time profiles of SOA predictions at sites with maximum difference in 24-h averaged SOA predictions with or without consideration of aqueous-phase organic chemistry in clouds on 3 August 2004 in the northern Gulf of Maine (a) and 4 August 2004 in the northern Gulf of Maine (b).

chemistry is reduced to approximately $18 \%$ larger than that without aqueous-phase organic chemistry for Scenario 1. In addition, locations influenced strongly by monoterpene emissions may not be necessarily exposed to cloud processing. These two reasons are believed to explain a much smaller relative SOA increase in the three-dimensional model simulation compared to the zero-dimensional model simulations if SOA formation from aqueous-phase organic chemistry in clouds is considered.

Figure 5 indicates the time series of the SOA predictions with or without consideration of SOA formation from the aqueous-phase organic chemistry in clouds at the two sites that have the maximum difference in 24-h average SOA predictions for 3 August and 4 August 2004, respectively. At these two sites, the relative difference in SOA prediction with or without consideration of SOA formation from aqueousphase organic chemistry is as high as $36 \%$ and $43 \%$, respectively, indicating that accounting for SOA formation from aqueous-phase organic chemistry in clouds could be important at these locations. As shown in Fig. 5, the increase of SOA formation by considering aqueous-phase organic chemistry is due mainly to the increase of SOA formation from S4 and S5, which is again attributed to the aqueous-phase ox- idation of S5 and S10, which are surrogates for oxidation products of monoterpenes. This implies that the increase of SOA formation with consideration of aqueous-phase organic chemistry in clouds is due to the cloud processing of monoterpene oxidation products. These two sites are influenced by transport of monoterpenes or monoterpene oxidation products from the New England region. In addition, these two sites are also exposed to resolved clouds almost throughout the entire episode. Therefore, SOA formation from aqueous-phase organics can be important when a site is influenced by biogenic monoterpene emissions or products and also exposed to cloud processing for significant time periods.

An interesting feature of Fig. 5 is that the increase in the difference in the SOA predictions usually occurs during afternoon hours. This is a direct result of $\mathrm{OH}$ being the only oxidant considered in the aqueous phase. Typically, $\mathrm{OH}$ concentrations in clouds reach a peak in the early afternoon (Ervens et al., 2003), which facilitates the conversion of organic compounds in cloud droplets and further formation of SOA. Differences in SOA prediction with or without consideration of SOA formation through cloud processing was also examined for three specific sites: Thompson Farm, New Hampshire (semi-rural), Boston, Massachusetts (urban), and Appledore Island, Maine (marine area influenced by continental outflow) and was found to be approximately $5 \%$. These sites were chosen because of different emissions patterns and relative influences of primary emissions and secondary chemistry.

Figure 6 indicates additional SOA formation due to the incloud organic chemistry at layer 14 of the modeling domain. In addition, for comparative purposes, the total SOA predictions from the simulations with consideration of SOA formation from aqueous-phase organic chemistry are also shown for layer 14. The CMAQ modeling domain includes 21 vertical layers using a $\sigma$-pressure system extending from the surface to $10000 \mathrm{~Pa}$. Although the actual altitude for layer 14 varies, according to the U.S. standard atmosphere, layer 14 corresponds to the altitude between 1800 and $2300 \mathrm{~m}$. Layer 14 thus roughly represents the lower free troposphere. The maximum additional SOA formation due to aqueous-phase organic chemistry in clouds is $0.25 \mu \mathrm{g} \mathrm{m}^{-3}$ for both days, only slightly smaller than the maximum difference at the surface. Similar to the surface layer, the increase of SOA predictions is due primarily to cloud processing of gas-phase monoterpene oxidation products, indicating the potential of SOA formation from an aqueous-phase mechanism involving biogenic precursors as suggested by Heald et al. (2006). Averaged across the modeling domain for layer 14 , the relative difference in SOA predictions is $19 \%$ and $15 \%$, respectively for 3 August and 4 August, which is much larger than the relative difference at the surface layer. This is due to the fact that less SOA is predicted to form from gas-phase oxidation of organic compounds in the free troposphere (Heald et al., 2005). 


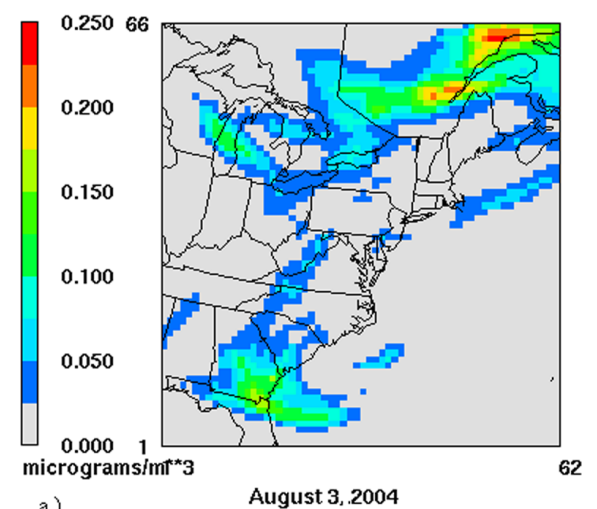

a.)
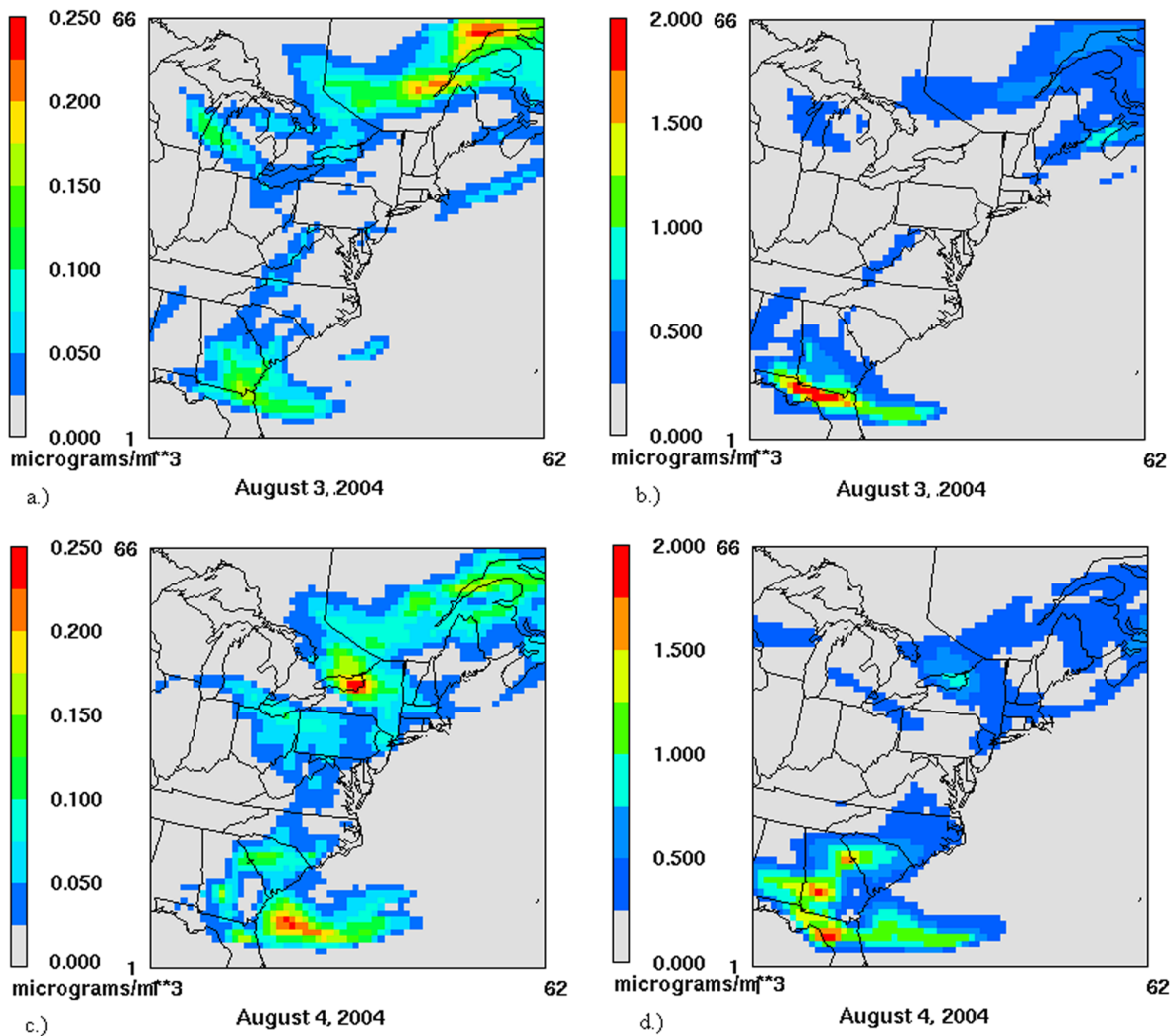

Fig. 6. Spatial distribution of SOA predictions of layer 14: (a) difference in 24-h average SOA prediction on 3 August 2004, with/without aqueous-phase organic chemistry simulation in clouds; (b) 24-h average SOA prediction on 3 August 2004, with aqueous-phase organic chemistry simulation in clouds; (c) difference in 24-h average SOA prediction on 4 August 2004, with/without aqueous-phase organic chemistry simulation in clouds; (d) 24-h average SOA prediction on 4 August 2004, with aqueous-phase organic chemistry simulation in clouds.

\section{Discussion}

An aqueous-phase chemistry mechanism was developed to study the potential of SOA formation in cloud droplets. The AqChem mechanism incorporates a compact treatment of hydrogen-oxygen and sulfur chemistry but treats in more detail organic chemistry based on recent work of Aumont et al. (2000), Ervens et al. (2004), and Lim et al. (2005) in an effort to represent the potential irreversible organic reactions that may lead to low-volatility products. AqChem was coupled to the existing CACM mechanism and MPMPO module. Zero-dimensional model simulations indicated that consideration of SOA formation from organic chemistry in cloud droplets could lead to increases of SOA prediction by approximately $27 \%$ and $7 \%$ for a rural scenario with strong monoterpene emissions and an urban scenario with strong aromatic compound emissions, respectively. Threedimensional model simulations showed an average of $8-9 \%$ increase in the SOA predictions at the surface when the aqueous-phase organic chemistry in clouds is considered. However, the relative increase of SOA prediction at certain locations could be much higher than the domain-wide, average surface increase. Overall, based on the proposed AqChem, the modeling work in this study suggests that SOA formation due to aqueous-phase organic chemistry in clouds could be important at locations that are strongly influenced by monoterpene emissions and where clouds are present for significantly long time, especially in the afternoon when photochemistry is strongest.

A key limitation of this study is the simple treatment of organic reactions in the aqueous phase for organic compounds of carbon number greater than four. The assumption that aldehydes of carbon number greater than four fully convert to the corresponding carboxylic acids may overestimate the yield of carboxylic acids from the reaction of aldehydes with $\mathrm{OH}$ in clouds. Thus, the results from the current study represent an upper-bound estimate of SOA formation via this route in clouds. Herrmann et al. (2005) presented a much more detailed treatment of organic chemistry in their aqueous-phase chemistry mechanism, although such a mechanism is probably too complex for incorporation into three-dimensional models. More laboratory studies are also needed to characterize the mechanism of aqueous-phase reactions, especially for intermediate gas-phase oxidation products, which are sol- 
uble in water and have the potential to lead to low-volatility products.

Another limitation is the lack of treatment of potentially reversible oligomerization processes, especially for small molecules like glyoxal and methyl glyoxal (Hastings et al., 2005; Loeffler et al., 2006). These processes are not included because of a current lack of a quantitative description.

In addition, future studies need to consider the aqueousphase processing of organic compounds in deliquescent particles. Unlike short-lived clouds, deliquescent particles provide additional time for processing organic compounds and could be more likely to lead to SOA, despite having significantly smaller water contents (Claeys et al., 2004). To treat similar processes in deliquescent particles, the present multiphase mechanism needs to be revised to consider interactions of different ions and molecules in the particles.

Despite these limitations, the method used in this study provides a framework to study SOA formation due to aqueous-phase processing of organic compounds and underscores the potential of SOA formation via cloud processing of organic compounds. New findings of aqueous-phase organic chemistry should be incorporated into this framework when such quantitative information becomes available.

Acknowledgements. Financial support for this work was provided by the Electric Power Research Institute and by the Office of Oceanic and Atmospheric Research of the National Oceanic and Atmospheric Administration under AIRMAP grants NA03OAR4600122 and NA04OAR4600154. We thank H. Mao for provision of model input files for the eastern United States. The development and availability of KPP 2.1 by A. Sandu and his coworkers is greatly appreciated.

Edited by: A. Nenes

\section{References}

Altieri, K. E., Carlton, A. G., Lim, H. J., Turpin, B. J., and Seitzinger, S. P.: Evidence for oligomer formation in clouds: Reactions of isoprene oxidation products, Environ. Sci. Technol., 40, 4956-4960, 2006.

Aumont, B., Madronich, S., Bey, I., and Tyndall, G. S.: Contribution of secondary VOC to the composition of aqueous atmospheric particles; a modeling approach, J. Atmos. Chem., 35, 59$75,2000$.

Blando, J. D., Porcja, R. J., Li, T. H., Bowman, D., Lioy, P. J., and Turpin, B. J.: Secondary formation and the Smoky Mountain organic aerosol: An examination of aerosol polarity and functional group composition during SEAVS, Environ. Sci. Technol., 32, 604-613, 1998.

Blando, J. D. and Turpin, B. J.: Secondary organic aerosol formation in cloud and fog droplets: a literature evaluation of plausibility, Atmos. Environ., 34, 1623-1632, 2000.

Byun, D. W. and Ching, J. K. S.: Science algorithms of the EPA Models-3 Community Multiscale Air Quality (CMAQ) modeling system, Report EPA/600/R-99/030, Office of Research and
Development, United States Environmental Protection Agency, Washington, DC, 1999.

Carlton, A. G., Turpin, B. J., Lim, H. J., Altieri, K. E., and Seitzinger, S.: Link between isoprene and secondary organic aerosol (SOA): Pyruvic acid oxidation yields low volatility organic acids in clouds, Geophys. Res. Lett., 33, L06822, doi:10.1029/2005GL025374, 2006.

Charlson, R. J., Schwartz, S. E., Hales, J. M., Cess, R. D., Coakley Jr., J. A., Hansen, J. E., and Hofmann D. J.: Climate forcing by anthropogenic aerosols, Science, 255, 423-430, 1992.

Chen, J. and Griffin, R. J.: Modeling secondary organic aerosol formation from oxidation of $\alpha$-pinene, $\beta$-pinene, and d-limonene, Atmos. Environ., 39, 7731-7744, 2005.

Chen, J., Mao, H., Talbot, R. W., and Griffin, R. J.: Application of the CACM and MPMPO modules using the CMAQ model for the eastern United States, J. Geophys. Res., 111, D23S25, doi:10.1029/2006JD007603, 2006.

Claeys, M., Wang, W., Ion, A. C., Kourtchev, I., Gelencser, A., and Maenhaut, W.: Formation of secondary organic aerosols from isoprene and its gas-phase oxidation products through reaction with hydrogen peroxide, Atmos. Environ., 38, 4093-4098, 2004.

Daescu, D., Sandu, A., and Carmichael, G. R.: Direct and adjoint sensitivity analysis of chemical kinetic systems with KPP: II Numerical validations and applications, Atmos. Environ., 37, 50975114, 2003.

Damian, V., Sandu, A., Damian, M., Potra, F., and Carmichael, G. R.: The kinetic preprocessor KPP - A software environment for solving chemical kinetics, Comput. Chem. Eng., 26, 1567-1579, 2002.

Decesari, S., Facchini, M. C., Fuzzi, S., and Tagliavini, E.: Characterization of water-soluble organic compounds in atmospheric aerosol: A new approach, J. Geophys. Res., 105, 1481-1489, 2000.

Dutot, A., Rude, J., and Aumont, B.: Neural network method to estimate the aqueous rate constants for the $\mathrm{OH}$ reactions with organic compounds, Atmos. Environ., 37, 269-276, 2003.

Ervens, B., George, C., Williams, J. E., et al.: CAPRAM 2.4 (MODAC mechanism): An extended and condensed tropospheric aqueous phase mechanism and its application, J. Geophys. Res., 108, 4426, doi:10.1029/2002JD002202, 2003.

Ervens, B., Feingold, G., Frost, G. J., and Kreidenweis, S. M.: A modeling study of aqueous production of dicarboxylic acids: 1 . Chemical pathways and speciated organic mass production, J. Geophys. Res., 109, D15205, doi:10.1029/2003JD004387, 2004.

Gelencser, A. and Varga, Z.: Evaluation of the atmospheric significance of multiphase reactions in atmospheric secondary organic aerosol formation, Atmos. Chem. Phys., 5, 2823-2831, 2005, http://www.atmos-chem-phys.net/5/2823/2005/.

Griffin, R. J., Cocker III, D. R., Flagan, R. C., and Seinfeld, J. H.: Organic aerosol formation from the oxidation of biogenic hydrocarbons, J. Geophys. Res., 104, 3555-3567, 1999.

Griffin, R. J., Dabdub, D., and Seinfeld, J. H.: Secondary organic aerosol, 1. Atmospheric chemical mechanism for production of molecular constituents, J. Geophys. Res., 107, 4332, doi:10.1029/2001JD000541, 2002.

Griffin, R. J., Dabdub, D., and Seinfeld, J. H.: Development and initial evaluation of a dynamic species-resolved model for gasphase chemistry and size-resolved gas/particle partitioning associated with secondary organic aerosol formation, J. Geophys. 
Res., D05304, doi:10.1029/2004JD005219, 2005.

Griffin, R. J., Nguyen, K., Dabdub, D., and Seinfeld, J. H.: A coupled hydrophobic-hydrophilic model for predicting secondary organic aerosol formation, J. Atmos. Chem., 44, 171-190, 2003.

Hastings, W. P., Koehler, C. A., Bailey, E. L., and DeHaan, D. O.: Secondary organic aerosol formation by glyoxal hydration and oligomer formation: Humidity effects and equilibrium shifts during analysis, Environ. Sci. Technol., 39, 8728-8735, 2005.

Heald, C. L., Jacob, D. J., Park, R. J., Russell, L. M., Huebert, B. J., Seinfeld, J. H., Liao, H., and Weber, R. J.: A large organic aerosol source in the free troposphere missing from current models, Geophys. Res. Lett., L18809, doi:10.1029/2005GL023831, 2005.

Heald, C. L., Jacob, D. J., Turquety, S., et al.: Concentrations and sources of organic carbon aerosols in the free troposphere over North America, J. Geophys. Res., 111, D23S47, doi:1029/2006JD007705, 2006.

Herrmann, H., Tilgner, A., Barzaghi, P., Majdik, Z., Gligorovski, S., Poulain, L., and Monod, A.: Towards a more detailed description of tropospheric aqueous phase organic chemistry: CAPRAM 3.0, Atmos. Environ., 39, 4351-4363, 2005.

Jang, M., Czoschke, N. M., Lee, S., and Kamens, R. M.: Heterogeneous atmospheric aerosol production by acid-catalyzed particlephase reactions, Science, 298, 814-817, 2002.

Jones, A., Roberts, L., and Slingo, A.: A climate model study of indirect radiative forcing by anthropogenic sulphate aerosols, Nature, 370, 450-453, 1994.

Kalberer, M., Paulsen, D., Sax, M., et al.: Identification of polymers as major components of atmospheric organic aerosols, Science, 303, 1659-1662, 2004.

Kanakidou, M., Seinfeld, J. H., Pandis, S. N., et al.: Organic aerosol and global climate modeling: a review, Atmos. Chem. Phys., 5, 1053-1123, 2005,

http://www.atmos-chem-phys.net/5/1053/2005/.

Kawamura, K. and Ikushima, K.: Seasonal changes in the distribution of dicarboxylic acids in the urban atmosphere, Environ. Sci. Technol., 27, 2227-2235, 1993.

Lim, H. J., Carlton, A. G., and Turpin, B. J.: Isoprene forms secondary organic aerosol through cloud processing: model simulations, Environ. Sci. Technol., 39, 4441-4446, 2005.

Loeffler, K. W., Koehler, C. A., Paul, N. M., and DeHaan, D. O.: Oligomer formation in evaporating aqueous glyoxal and methyl glyoxal solutions, Environ. Sci. Technol., 40, 6318-6323, 2006.

Malm, W. C.: Atmospheric haze: Its sources and effects on visibility in rural areas of the continental United States, Environ. Monit. Assess., 12, 203-225, 1989.

Mao, H., Talbot, R., Troop, D., Johnson, R., Businger, S., and Thompson, A. M.: Smart balloon observations over the North Atlantic: $\mathrm{O}_{3}$ data analysis and modeling, J. Geophys. Res., 111, D23S56, doi:10.1029/2005JD006507, 2006.

Martin, L. R. and Good, T. W.: Catalyzed oxidation of sulfur dioxide in solution: the iron-manganese synergism, Atmos. Environ., 25A, 2395-2399, 1991.

Murphy, D. M., Thomson, D. S., and Mahoney, M. J.: In situ measurements of organics, meteoritic material, mercury, and other elements in aerosols at 5 to 19 kilometers, Science, 282, 16641669, 1998.

Odum, J. R., Hoffmann, T., Bowman, F., Collins, D., Flagan, R. C., and Seinfeld, J. H.: Gas/particle partitioning and secondary organic aerosol yields, Environ. Sci. Technol., 30, 2580-2585, 1996.

Pandis, S. N. and Seinfeld, J. H.: Sensitivity analysis of a chemical mechanism for aqueous-phase atmospheric chemistry, J. Geophys. Res., 94, 1105-1126, 1989.

Pankow, J. F.: An absorption model of the gas/aerosol partitioning involved in the formation of secondary organic aerosol, Atmos. Environ., 28, 189-193, 1994.

Pope, C. A. and Dockery, D. W.: Health effects of fine particulate air pollution: Lines that connect, J. Air. Waste Manage. Assoc., 56, 709-742, 2006.

Pun, B. K., Griffin, R. J., Seigneur, C., and Seinfeld, J. H.: Secondary organic aerosol: II: Thermodynamic model for gas/particle partitioning of molecular constituents, J. Geophys. Res., 107, 4333, doi:10.1029/2001JD000542, 2002.

Sandu, A., Daescu, D., and Carmichael, G. R.: Direct and adjoint sensitivity analysis of chemical kinetic systems with KPP: I Theory and software tools, Atmos. Environ., 37, 5083-5096, 2003.

Sandu, A., Verwer, J. G., Blom, J. G., Spee, E. J., Carmichael, G. R., and Potra, F. A.: Benchmarking stiff ODE solvers for atmospheric chemistry problems II: Rosenbrock methods, Atmos. Environ., 31, 3459-3472, 1997.

Schwartz, S. E.: Mass-transport considerations pertinent to aqueous-phase reactions of gases in liquid-water clouds, in: Chemistry of Multiphase Atmospheric Systems, edited by: Jaeschke, W., Springer, New York, 1986.

Sorooshian, A., Varutbangkul, V., Brechtel, F. J., et al.: Oxalic acid in clear and cloudy atmospheres: Analysis of data from International Consortium for Atmospheric Research on Transport and Transformation 2004, J. Geophys. Res., 111, D23S45, doi:10.1029/2005JD006880, 2006.

Sorooshian, A., Lu, M. L., Brechtel, F. J., Jonsson, H., Feingold, G., Flagan, R. C., and Seinfeld, J. H.: On the source of organic acid aerosol layers above clouds, Environ. Sci. Technol., 41, 46474654, 2007.

Tulet, P., Grini, A., Griffin, R. J., and Petitcol, S.: ORILAM-SOA: A computationally efficient model for predicting secondary organic aerosols in 3D atmospheric models, J. Geophys. Res., D23208, doi:10.1029/2006JD007152, 2006.

Turpin, B. J., Saxena, P., and Andrews, E.: Measuring and simulating particulate organics in the atmosphere: Problems and prospects, Atmos. Environ., 34, 2983-3013, 2000.

Warneck, P.: The relative importance of various pathways for the oxidation of sulfur dioxide and nitrogen dioxide in sunlit continental fair weather clouds, Phys. Chem. Chem. Phys., 1, 54715483, 1999.

Warneck, P.: In-cloud chemistry opens pathway to the formation of oxalic acid in the marine atmosphere, Atmos. Environ., 37, 2423-2427, 2003.

Yao, X., Fang, M., and Chan, C. K.: Size distributions and formation of dicarboxylic acids in atmospheric particles, Atmos. Environ., 36, 2099-2107, 2002.

Zaveri, R.: Development and evaluation of a comprehensive tropospheric chemistry model for regional and global application, dissertation, Virginia Polytechnic Institute and State University, Blacksburg, Virginia, 1997. 